Journal Club

Editor's Note: These short, critical reviews of recent papers in the Journal, written exclusively by graduate students or postdoctoral fellows, are intended to summarize the important findings of the paper and provide additional insight and commentary. For more information on the format and purpose of the Journal Club, please see http://www.jneurosci.org/misc/ifa_features.shtml.

\title{
Stimulus-Driven Brain Oscillations in the Alpha Range: Entrainment of Intrinsic Rhythms or Frequency-Following Response?
}

\author{
(1)Christian Keitel, ${ }^{1 *}$ (1)Cliodhna Quigley, ${ }^{2 *}$ and $\odot$ Philipp Ruhnau ${ }^{3}$ \\ ${ }^{1}$ Institute of Neuroscience and Psychology, University of Glasgow, G12 8QB Glasgow, United Kingdom, ${ }^{2}$ Cognitive Neuroscience \\ Laboratory, German Primate Center, 37077 Göttingen, Germany, and ${ }^{3}$ Center for Mind/Brain Sciences (CIMeC), Università degli \\ Studi di Trento, 38060 Mattarello (Trento), Italy \\ Review of Spaak et al.
}

Human brain activity is rich in rhythms of various characteristic frequencies. The last few decades have seen an increase in their use as an explanatory means, with a vast literature describing manifold correlations between dynamics of brain rhythms and behavioral performance in perceptual and cognitive tasks involving attention, memory, and language. More recently, the desire to study the causal role of neural rhythms in stimulus processing and corresponding performance has raised interest in externally entraining these rhythms.

The endogenous alpha rhythm $(8-12$ $\mathrm{Hz}$ ) is one of the few neural phenomena

Received May 12, 2014; revised June 11, 2014; accepted June 26, 2014.

The data mentioned in this Journal Club were recorded at the Institute of Psychology, University of Leipzig, Germany, under supervision of Matthias M. Müller and supported by the Deutsche Forschungsgemeinschaft graduate program "Function of Attention in Cognition" (GRK-1182). Publication was supported by the Wellcome Trust $(098433,098434)$. We thank Nathan Weisz and Joachim Gross for helpful comments on the manuscript, Gregor Thut for discussion, and Anne Keitel for proofreading.

${ }^{*}$ C.K. and C.Q. contributed equally.

This article is freely available online through the J Neurosci Author Open Choice option.

Correspondence should be addressed to the following: Christian Keitel, Institute of Neuroscience and Psychology, University of Glasgow, 58 Hillhead Street, G12 8QB Glasgow, United Kingdom, E-mail: Christian.Keitel@glasgow.ac.uk; Cliodhna Quigley, Cognitive Neuroscience Laboratory, German Primate Center, Kellnerweg 4, 37077 Göttingen, Germany, E-mail: CQuigley@dpz.eu; or Philipp Ruhnau, Center for Mind/Brain Sciences (CIMeC), Università degli Studi di Trento, via delle Regole, 101, 38060 Mattarello (Trento), Italia, E-mail: Philipp.Ruhnau@unitn.it.

DOI:10.1523/JNEUROSCI.1904-14.2014

Copyright $\odot 2014$ the authors $\quad 0270-6474 / 14 / 3410137-04 \$ 15.00 / 0$ visible in raw electroencephalographic (EEG) or magnetoencephalographic (MEG) recordings because of its large-amplitude sinusoidal waveform. Subdivisions of the alpha rhythm based on cortical origin and/or functional role have been described, but the most prominent is the parietooccipital alpha rhythm. Current hypotheses ascribe this alpha rhythm an active role in human visual perception. For example, detection of visual stimuli has been shown to vary with the power (Thut et al., 2006) and relative phase of ongoing alpha oscillations (Busch et al., 2009).

In a recent study, Spaak et al. (2014) investigated the role of neural oscillations in visual stimulus perception. To do so, they entrained ongoing cortical activity locally (i.e., unilaterally) by presenting a train of 16 white squares rhythmically at a rate of $10 \mathrm{~Hz}$ in one visual hemifield and arrhythmically in the other. Subjects had to detect a lowcontrast target presented at varying intervals after flicker offset. This allowed Spaak et al. (2014) to disentangle the effects of rhythmic stimulation on behavior while using MEG to measure the oscillatory brain response entrained by the stimulation. In accordance with a recent line of research (Mathewson et al., 2012; de Graaf et al., 2013), the authors reasoned that their choice of flicker frequency within the human al- pha range would naturally lead to an entrainment of endogenous alpha oscillations.

In parallel, over the past two decades, another line of research has used rhythmic visual stimulation to drive neural oscillations with frequencies within and outside the alpha band. The associated method of frequency tagging is based on the fact that periodic visual stimulation will elicit a periodic neural response, more broadly known as the steady-state response (SSR). Because the experimenter knows which stimulation frequencies were used, SSRs can easily be isolated from the EEG or MEG by spectral decomposition. The main advantage of this method is that multiple, simultaneously presented stimuli can be tagged with different frequencies, thus allowing analyses of brain responses to each individual element of a multistimulus display. SSRs have been widely used to study cognitive influences on ongoing visual stimulus processing (Kim et al., 2007; Andersen and Müller, 2010; Keitel et al., 2013).

Frequency tagging does not assume any relation between the SSR and the preferred oscillation frequency of the participating neuronal population. In fact, frequency-following responses occur for a wide range of stimulation frequencies, many of which have shown similar susceptibility to experimental manipulations. Frequency tagging is thus seen as a 

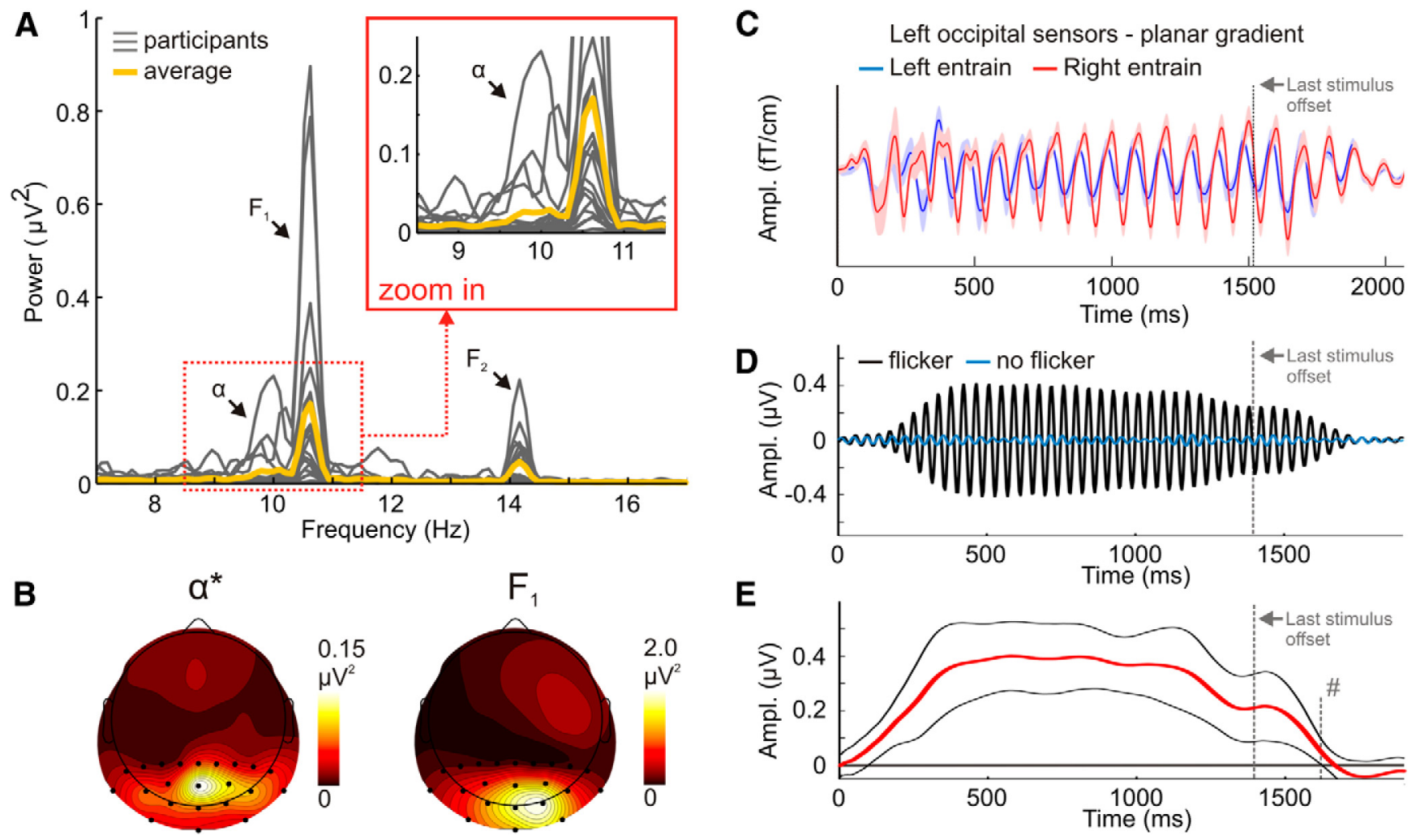

Figure 1. A, Condition-averaged power spectra of individual participants ( $N=14$; gray lines) based on data from Keitel et al. (2013). Heavy yellow lines depict the Grand Average spectrum. Data were preprocessed as described in the original study but epochs were subjected to Fourier transforms before averaging to retain oscillatory neural activity not phase-locked to stimulation. Narrow-band peaks at $\mathrm{F}_{1}=10.7 \mathrm{~Hz}$ and $\mathrm{F}_{2}=14.2 \mathrm{~Hz}$ correspond to stimulation frequencies in left and right hemifields, respectively. Inset magnifies $9-11$ $\mathrm{Hz}$. Broader peaks, here denoted with $\alpha$, are visible adjacent to $\mathrm{F}_{1} \cdot \boldsymbol{B}, \alpha$ subband $\left(10 \pm 0.25 \mathrm{~Hz}\right.$; denoted by $\left.{ }^{*}\right)$ power and $\mathrm{F}_{1}$ SSR power topographies for the five participants exhibiting maximal $\alpha^{*}$ power. Black dots indicate the electrode cluster from which spectra were calculated. Note different scales. C, Adapted from Spaak et al. (2014) (with permission). Grand Average event-related field elicited by a left (blue) or right hemifield (red) $10 \mathrm{~Hz}$ stimulus train. The oscillation reverberates after the last stimulus offset (dashed gray line). $\boldsymbol{D}$, Grand Average event-related potential ( $N=19$; electrodes $\mathrm{POz}, 01,0 \mathrm{z}, 02$, Iz; two-pass finite impulse response filter: 256-point Blackman window, pass band $22-26 \mathrm{~Hz}$ ) derived from data of Porcu et al. (2014). Here, a visual stimulus train presented at a rate of $24 \mathrm{~Hz}$ for $\sim 1.4$ s elicited an SSR (black line) that reverberates after offset of the last stimulus. Blue line depicts ERP from a condition without rhythmic visual stimulation. Last stimulus offset is at $1396 \mathrm{~ms}$ (dashed gray line). $\boldsymbol{E}$, Hilbert amplitude difference time course (heavy red line) derived from waveforms in $\boldsymbol{D}$. Waveform envelopes were calculated by means of Hilbert transforms for each electrode and participant, then averaged for flicker-present and flicker-absent conditions. Subtracting flicker-absent from flicker-present yielded the depicted time course. Black lines illustrate upper and lower 95\%-confidence limits of a running $t$ test against zero (two-tailed). At \# (1635 ms; for a stricter 99\%-confidence limit: $1615 \mathrm{~ms}$ ), the lower bound crosses zero, indicating the oscillation's decay.

means to investigate visual processing without interfering with it, i.e., to "wiretap" visual perception. In contrast, the notion of alpha entrainment through rhythmic visual stimulation as used by Spaak et al. (2014) assumes that the driven brain oscillation is functionally identical to the endogenous alpha rhythm. Given alpha's active role in visual perception, its entrainment is understood as controlling or "hijacking" perceptual processes.

These two different perspectives have been more or less independently developed and, to our knowledge, they have not yet been systematically compared. Can SSRs with frequencies in the alpha band simply be explained by alpha entrainment? Clearly, these alpha SSRs should exhibit functional characteristics similar to those of the endogenous alpha rhythm. But here experimental observations give rise to a strong discrepancy: the assump- tion of alpha entrainment predicts that the amplitude of the driven brain response will exhibit alpha-typical modulations by top-down factors such as spatial attention. Whereas clear reductions in endogenous alpha amplitude have been found at recording sites contralateral to an attended stimulus location (Thut et al., 2006), SSR amplitude reliably increases when the driving stimulus' location is attended, regardless of whether or not the stimulation frequency is in the alpha range (Keitel et al., 2013).

It is this inconsistency that has led us to ask whether alpha entrainment follows immediately from using alpha band stimulation frequencies. In the following, we compare the results of Spaak et al. (2014) to data from a recent frequency tagging study (Keitel et al., 2013) featuring a similarly lateralized stimulation paradigm to establish the resemblance of entrained al- pha with an alpha-band SSR. We go on to review arguments brought forward by Spaak et al. (2014) that their measured oscillation indeed reflects alpha entrainment rather than an SSR. We conclude that the causal connection between rhythmic stimulation and the alpha rhythm is not straightforward and that unambiguous classification of stimulus-driven brain responses as entrained endogenous versus frequency-following is difficult and warrants further research.

In their study, Spaak et al. (2014) convincingly demonstrate that the $10 \mathrm{~Hz}$ entrainment occurs locally, in the sense that the cortical response measured likely arises from generators in early visual cortices contralateral to the rhythmically stimulated hemifield (Spaak et al., 2014, their Fig. 4). The entrained response is further local in terms of frequency; timefrequency representations in Spaak et al.'s 
(2014) Figure 3 show constant narrowband responses at the stimulation frequency. In comparison, the $10.7 \mathrm{~Hz}$ SSR driven by a lateral left-hemifield stimulus in Keitel et al. (2013) exhibits the same localness in both right-lateralized cortical generation and frequency.

Interestingly, the spectral representation of the EEG recorded during rhythmic visual presentation at $10.7 \mathrm{~Hz}$ was found to contain broad peaks in the $9-10 \mathrm{~Hz}$ range, indicative of alpha activity, in addition to the narrow-band peaks that corresponded to the stimulation frequencies (Fig. 1A). A topographical representation of alpha activity taken from the five participants who exhibited highest amplitudes in the 9-10 $\mathrm{Hz}$ range showed a central parietooccipital maximum. Figure $1 B$ illustrates that this topography differed from the $10.7 \mathrm{~Hz}$ SSR amplitude distribution of the same group of participants. This observation supports the notion of the SSR arising from locally entrained oscillators, in contrast with the co-occurring endogenous alpha activity, which likely originates from generators not entrained by the stimulation.

The most compelling evidence for alpha entrainment in Spaak et al. (2014) are the behavioral effects of the stimulation. In their paradigm, the target was presented at different latencies after the flashing squares, and therefore at specific phases of the rhythm entrained by one hemifield of their display. They found a rhythmic modulation of behavior: participants performed better when the target appeared anti-phase to the preceding rhythmical stimulation and worse when the target appeared in-phase. These effects of rhythmical presentation persisted for at least two to three alpha cycles.

Was this $10 \mathrm{~Hz}$ periodicity in detection performance due to the endogenous alpha influence reported in earlier studies (Busch et al., 2009), or could it be a previously unknown and unreported aspect of the SSR? Spaak et al. (2014) explicitly rejected SSRs as an alternative explanation on the grounds that stimulus-driven oscillations continued for a few cycles after stimulus offset, which they considered indicative of a reverberation of entrained alpha oscillators (Fig. 1C). Data on SSR behavior after stimulus offset is lacking and a model of visual SSR generation actually contradicts a reverberation (Capilla et al., 2011). Nevertheless, a recently collected dataset allowed the analysis of a $24 \mathrm{~Hz}$ (above alpha-band) visual SSR around stimulus offset (Porcu et al., 2014). The resulting SSR reverberation (Fig. $1 D, E$ ) cannot be accounted for by an entrainment of intrinsic alpha generators, as the frequency is out of the alpha range. Although the experiment was not designed to test this hypothesis, this finding indicates that SSRs might generally show a reverberation after stimulus offset.

However, assuming a close link between the reverberation and behavior, periodic modulations of behavioral performance driven by periodic stimulation regimes might be observed over a wider range of tagging frequencies (Jokeit, 1990). This presents a clear and important challenge to the assumed causal neutrality of rhythmic stimulation in SSR paradigms and strongly motivates further research. Experiments have yet to be performed using a wide range of stimulation frequencies outside the alpha band, but early results are intriguing. As de Graaf et al. (2013) recently demonstrated, both a 5.3 $\mathrm{Hz}$ and $10.6 \mathrm{~Hz}$ visual stimulation lead to an alpha-band periodicity in behavioral performance.

Ultimately, this raises the question of whether tagging stimuli with alpha-band frequencies constitutes a confound in SSR research. Three challenges in particular come to mind. First, SSR and eventrelated potential approaches are founded on the assumption that task-irrelevant brain activity occurs independently in time with respect to stimulus and task timing. Averaging brain responses to multiple repetitions of the same stimulus attenuates the contribution of this "noise." However, transient stimulation or a specific task can affect alpha oscillations in a temporally consistent manner (for discussion, see van Dijk et al., 2010). As such, the choice of a stimulation frequency in the alpha range may result in the experimenter unwittingly measuring a mixture of modulations of an externally driven SSR and an endogenous alpha oscillator induced by a stimulus or task. Second, in the earliest characterizations of visual SSR, $10 \mathrm{~Hz}$ was deemed to be special in that it generated the largest amplitude response and exhibited resonance-like properties (Regan, 1966). This must, however, be qualified by the clear evidence Regan (1966) found for the independence of SSR and endogenous alpha oscillations $-10 \mathrm{~Hz}$ is not necessarily parietooccipital alpha. In fact, recent modeling suggests that stimulation at around 10 $\mathrm{Hz}$ or its harmonics rather gives rise to unique nonlinearities that indicate interactions between visual stimulation and endogenous alpha (Roberts and Robinson, 2012). Finally, the behavioral effect reported by Spaak et al. (2014) raises the most important challenge. Whether or not the effect is due to entrainment of endogenous oscillations or to SSRs, the point still stands that rhythmic stimulation influenced the visual perception of their subjects.

In conclusion, while Spaak et al. (2014) and others (Mathewson et al., 2012; de Graaf et al., 2013) treat stimulus-driven $10 \mathrm{~Hz}$ oscillations as the entrained alpha rhythm, SSR studies do not make a connection between their narrow-band evoked responses and the classical frequency bands delineating spontaneous neural rhythms. As a consequence, although these two concurrent lines of research use highly similar paradigms to elicit brain oscillations, they make disparate assumptions about the nature of the resulting oscillations. Put differently, one school of thought claims alpha generators can be hijacked by rhythmic stimulation, while the other believes that rhythmic stimulation can be used to wire-tap cortical activity. It is vital to resolve this discrepancy, as only the combination of both lines will allow for a clear interpretation of the role of neural rhythms in general, and the alpha rhythm in particular, in perception and cognitive function.

\section{References}

Andersen SK, Müller MM (2010) Behavioral performance follows the time course of neural facilitation and suppression during cued shifts of feature-selective attention. Proc Natl Acad Sci U S A 107:13878-13882. CrossRef Medline

Busch NA, Dubois J, VanRullen R (2009) The phase of ongoing EEG oscillations predicts visual perception. J Neurosci 29:7869-7876. CrossRef Medline

Capilla A, Pazo-Alvarez P, Darriba A, Campo P, Gross J (2011) Steady-state visual evoked potentials can be explained by temporal superposition of transient event-related responses. PLoS One 6:e14543. CrossRef Medline

de Graaf TA, Gross J, Paterson G, Rusch T, Sack AT, Thut G (2013) Alpha-band rhythms in visual task performance: phase-locking by rhythmic sensory stimulation. PLoS One 8:e60035. CrossRef Medline

Jokeit H (1990) Analysis of periodicities in human reaction times. Naturwissenschaften 77 : 289-291. CrossRef Medline

Keitel C, Andersen SK, Quigley C, Müller MM (2013) Independent effects of attentional gain control and competitive interactions on visual stimulus processing. Cereb Cortex 23: 940-946. CrossRef Medline

Kim YJ, Grabowecky M, Paller KA, Muthu K, Suzuki S (2007) Attention induces synchronization-based response gain in steady-state visual evoked potentials. Nat Neurosci 10:117-125. CrossRef Medline

Mathewson KE, Prudhomme C, Fabiani M, Beck DM, Lleras A, Gratton G (2012) Making 
waves in the stream of consciousness: entraining oscillations in EEG alpha and fluctuations in visual awareness with rhythmic visual stimulation. J Cogn Neurosci 24: 2321-2333. CrossRef Medline

Porcu E, Keitel C, Müller MM (2014) Visual, auditory and tactile stimuli compete for early sensory processing capacities within but not between senses. Neuroimage 97:224-235. CrossRef Medline

Regan D (1966) Some characteristics of average steady-state and transient responses evoked by modulated light. Electroencephalogr Clin Neurophysiol 20:238-248. CrossRef Medline

Roberts JA, Robinson PA (2012) Quantitative theory of driven nonlinear brain dynamics. Neuroimage 62:1947-1955. CrossRef Medline

Spaak E, de Lange FP, Jensen O (2014) Local entrainment of alpha oscillations by visual stimuli causes cyclic modulation of perception. J Neurosci 34:3536-3544. CrossRef Medline
Thut G, Nietzel A, Brandt SA, Pascual-Leone A (2006) Alpha-band electroencephalographic activity over occipital cortex indexes visuospatial attention bias and predicts visual target detection. J Neurosci 26:9494-9502. CrossRef Medline

van Dijk H, van der Werf J, Mazaheri A, Medendorp WP, Jensen O (2010) Modulations in oscillatory activity with amplitude asymmetry can produce cognitively relevant eventrelated responses. Proc Natl Acad Sci U S A 107:900-905. CrossRef Medline 